\title{
In-hospital mortality after hip fracture by treatment setting
}

\author{
Katie J. Sheehan PhD, Boris Sobolev PhD, Pierre Guy MD MBA, Lisa Kuramoto MSc, Suzanne N. Morin MD MSc, \\ Jason M. Sutherland PhD, Lauren Beaupre PhD, Donald Griesdale MD MPH, Michael Dunbar PhD MD, \\ Eric Bohm MD, Edward Harvey MD MSc; for The Canadian Collaborative Study of Hip Fractures*
}

\begin{abstract}
Background: Where patients with hip fracture undergo treatment may influence their outcome. We compared the risk of in-hospital death after hip fracture by treatment setting in Canada.

Methods: We examined all discharge abstracts from the Canadian Institute for Health Information with diagnosis codes for hip fracture involving patients 65 years and older who were admitted to hospital with a nonpathological first hip fracture between Jan. 1, 2004, and Dec. 31, 2012, in Canada (excluding Quebec). We compared the risk of in-hospital death, overall and after surgery, between teaching hospitals and community hospitals of various bed capacities, accounting for variation in length of stay.
\end{abstract}

Results: Compared with the number of deaths per 1000 admissions at teaching hospitals, there were an additional 3 (95\% confidence interval [Cl] 1-6), $14(95 \% \mathrm{Cl} 10-18)$ and $43(95 \% \mathrm{Cl}$ 35-51) deaths per 1000 admissions at large, medium and small community hospitals, respectively. For the risk of in-hospital death overall, the adjusted odds ratios (ORs) were 1.05 (95\% Cl 0.99-1.11), 1.16 (95\% Cl 1.09-1.24) and 1.44 (95\% Cl 1.31-1.57) at large, medium and small community hospitals, respectively, compared with teaching hospitals. For the risk of postsurgical death in hospital, the adjusted ORs were 1.06 (95\% Cl 1.00-1.13), 1.13 (95\% Cl 1.04-1.23) and 1.18 (95\% Cl $0.87-1.60)$ at large, medium and small community hospitals, respectively.

Interpretation: Compared with teaching hospitals, the risk of in-hospital death among patients with hip fracture was higher at medium and small community hospitals, and the risk of in-hospital death after surgery was higher at medium community hospitals. No differences were found between teaching and large community hospitals. Future research should examine the role of volume, demand and bed occupancy for observed differences.
$\mathrm{O}$ ne in 10 patients with hip fracture die during their hospital stay. ${ }^{1-3}$ The risk of death is associated with patient, injury and treatment characteristics. ${ }^{4,5}$ Treatment setting may also influence this risk. ${ }^{1,26-8}$ For example, advanced standards of anesthesia and surgery are associated with teaching hospitals, ${ }^{9,10}$ but there is inconsistent evidence for an association between teaching status and in-hospital death. $.^{9,11-13} \mathrm{Com}-$ paring teaching hospitals with community hospitals of different bed capacities may further our understanding of the risk of in-hospital death across treatment settings. Bed capacity is associated with factors of care delivery such as resources, treatment styles and standby capacity. ${ }^{14}$

Most patients undergo surgery to repair hip fracture. ${ }^{15}$ However, between $6 \%$ and $10 \%$ of patients do not receive surgery, in some cases because of death while waiting for surgery. ${ }^{16,17}$ To better understand the risk of in-hospital death by treatment setting, outcomes of both surgical and nonsurgical care should be considered. Therefore, we conducted this study to compare the risks of in-hospital death, overall and after surgery, between teaching hospitals and community hospitals of various bed capacities providing hip fracture care in Canada.

\section{Methods}

\section{Design, setting and population}

We obtained all discharge abstracts with diagnosis codes for hip fracture (International Classification of Diseases, ninth revision, code 820; and International Statistical Classification of Diseases and Related Health Problems, 10th revision, codes S72.00, S72.01, S72.09, S72.10, S72.19, S72.20) involving patients 65 years and older who were admitted to hospital with a nonpathological first hip fracture between Jan. 1, 2004, and Dec. 31, 2012, in Canada (except for the province of Quebec) from the Canadian Institute for Health Information (CIHI) Discharge Abstract Database. ${ }^{18}$ Multiple abstracts with the same patient identifier were combined into a single care episode using the CIHI rules for hospital transfers. ${ }^{19,20}$
Competing interests: See end of article.

This article has been peer reviewed.

Accepted: Aug. 9, 2016 Online: Oct. 17, 2016

Correspondence to: Katie Sheehan, sheehakj@ mail.ubc.ca; Boris Sobolev, boris.sobolev@ubc.ca

CMAJ 2016. DOI:10.1503/ cmaj.160522 
For estimating the risk of postsurgical death, we selected discharge abstracts with procedural codes for hip fracture surgery (Canadian Classification of Health Interventions codes $1 \mathrm{VA} 74^{\wedge} \wedge, 1 \mathrm{VA} 53^{\wedge \wedge}$, $1 \mathrm{VC} 74^{\wedge \wedge}$ and $1 \mathrm{SQ} 53^{\wedge \wedge}$; Canadian Classification of Diagnostic, Therapeutic and Surgical Procedures codes 9054, 9114, 9134, 9351, 9359, 9361, 9362, 9363, 9364 and 9369), a valid surgery date and a hospital stay of at least 1 day after surgery. We considered deaths on the day of surgery as intraoperative, and live hospital discharge on the day of surgery as clinically unjustifiable.

\section{Outcomes}

The primary outcome was in-hospital death identified by destination code in the discharge abstracts. The time to death was calculated as the number of days from the date of admission (counting the admission day) to the date of death, hospital discharge or 30 days, whichever came first. Postsurgical death referred to deaths on abstracts with a code for hip fracture surgery. The time to postsurgical death was calculated as the number of days from the date of surgery to the date of death, hospital discharge or 30 days, whichever came first. In the analysis of deaths without surgery, we calculated the time to death as the number of days from the date of admission (counting the admission day) to the date of death, surgery, hospital discharge or 30 days, whichever came first.

\section{Treatment setting}

We used the definitions of CIHI's Canadian Hospital Reporting Project to classify treatment setting. Members of the Association of Canadian Academic Healthcare Organizations were classified as

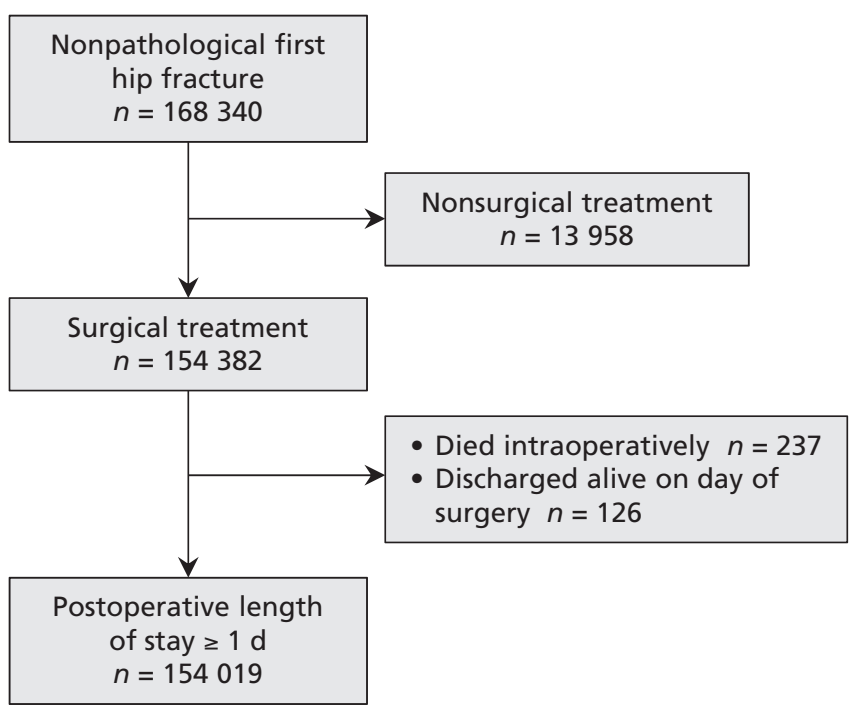

Figure 1: Study population. teaching hospitals; all other hospitals were community hospitals, grouped by the number of beds: small ( $<50$ beds), medium (50-199) and large $(\geq 200) .{ }^{21}$ Treatment setting at admission was a study variable in the analysis of in-hospital mortality, and treatment setting at surgery was a study variable in the analysis of postsurgical mortality.

\section{Statistical analysis}

We used the $\chi^{2}$ test to compare distributions of patient and care characteristics across treatment settings. We estimated daily rates of death overall and by treatment setting by dividing the number of corresponding events by the total number of inpatient days.

We estimated the cumulative incidence of death as a function of inpatient day, with live discharge as a competing event, assuming patients were at risk of in-hospital death only while they remained in hospital. ${ }^{22}$ We identified live discharges by the following destination codes: discharged home, discharge to home with support, or transferred to long-term care, palliative care, hospice or addiction treatment. We treated hospital stays that ended by transfer to acute care, discharges on the day after surgery and stays that exceeded 30 days as right-censored observations. ${ }^{20}$ In the analysis of deaths without surgery, surgery was an additional competing event. We used the Pepe-Mori 2-sample test ${ }^{22}$ and proportional odds regression models ${ }^{23}$ to test whether the cumulative incidences of death differed between teaching hospitals and community hospitals of various bed capacity. The differences were summarized by 30 -day risk differences and by odds ratios. ${ }^{24}$

In the regression analysis, the differences between treatment settings were adjusted for patient age, sex, fracture type, comorbidity (heart failure, chronic obstructive pulmonary disease, acute ischemic heart disease, hypertension, diabetes), ${ }^{25,26}$ province or territory, and the calendar period (2004-2006, 2007-2009 or 2010-2012), day (weekday v. weekend) and time of admission. We adjusted for type (internal fixation v. arthroplasty $)^{27}$ and timing of surgery in the analysis of postsurgical mortality. We conducted the competing-risk analysis using the pseudo-values method ${ }^{23}$ with $\mathrm{R}$ packages cmprsk, ${ }^{28}$ prodlim ${ }^{29}$ and geepack. ${ }^{30}$ The number of discharge abstracts was sufficient to detect a $1 \%$ increase in the risk of in-hospital death (from $7 \%$ to $8 \%$ ), and in the risk of postsurgical death (from 6\% to $7 \%$ ), with $90 \%$ power and a 2 -sided significance level of $5 \%$.

\section{Ethics approval}

The University of British Columbia Behavioural Research Ethics Board approved this study. 
Table 1: Patient and care characteristics of 168340 patients with a first hip fracture, by hospital type at admission

\begin{tabular}{|c|c|c|c|c|c|}
\hline Characteristic & \multicolumn{5}{|c|}{ Hospital type; no. (\%) of patients } \\
\hline \multicolumn{6}{|l|}{ Age at admission, yr } \\
\hline $65-74$ & $25314(15.0)$ & $9188(15.6)$ & $9921(14.4)$ & $4399(14.8)$ & $1503(16.1)$ \\
\hline $75-84$ & $65684(39.0)$ & 22768 (38.7) & 26991 (39.3) & $11629(39.2)$ & 3537 (37.9) \\
\hline $85-94$ & $68154(40.5)$ & $23548(40.0)$ & $28107(40.9)$ & $12093(40.7)$ & $3763(40.3)$ \\
\hline \multicolumn{6}{|l|}{ Fracture typet } \\
\hline Transcervical & $87248(51.8)$ & 30491 (51.9) & $35056(51.0)$ & $15644(52.7)$ & $5180(55.4)$ \\
\hline Pertrochanteric & $73093(43.4)$ & $25312(43.0)$ & $30360(44.2)$ & $12781(43.1)$ & $3798(40.7)$ \\
\hline Subtrochanteric & $7999(4.8)$ & $2996(5.1)$ & $3327(4.8)$ & $1259(4.2)$ & $365(3.9)$ \\
\hline \multicolumn{6}{|l|}{ Calendar year of admission } \\
\hline 2004 & $18927(11.2)$ & $6738(11.5)$ & $7352(10.7)$ & $3215(10.8)$ & $1115(11.9)$ \\
\hline 2009 & $18575(11.0)$ & $6501(11.1)$ & 7727 (11.2) & $3236(10.9)$ & $1031(11.0)$ \\
\hline 2010 & $18583(11.0)$ & $6383(10.9)$ & $7857(11.4)$ & $3280(11.0)$ & $971(10.4)$ \\
\hline 2011 & $18628(11.1)$ & $6505(11.1)$ & $7906(11.5)$ & $3278(11.0)$ & $914(9.8)$ \\
\hline 2012 & $19325(11.5)$ & $6648(11.3)$ & $8371(12.2)$ & $3436(11.6)$ & $870(9.3)$ \\
\hline \multicolumn{6}{|l|}{ Comorbidity $\ddagger$} \\
\hline Heart failure & $14250(8.5)$ & $5048(8.6)$ & $5709(8.3)$ & $2488(8.4)$ & $840(9.0)$ \\
\hline COPD & $9315(5.5)$ & $3117(5.3)$ & $3604(5.2)$ & $1816(6.1)$ & $651(7.0)$ \\
\hline Ischemic heart disease, acute & $10614(6.3)$ & $3928(6.7)$ & $4151(6.0)$ & $1864(6.3)$ & $550(5.9)$ \\
\hline Cardiac dysrhythmia & $16023(9.5)$ & $5526(9.4)$ & $6733(9.8)$ & $2758(9.3)$ & $848(9.1)$ \\
\hline Ischemic heart disease, chronic & $2955(1.8)$ & $1048(1.8)$ & $1240(1.8)$ & $481(1.6)$ & $147(1.6)$ \\
\hline Hypertension & $9982(5.9)$ & $3453(5.9)$ & $4254(6.2)$ & $1688(5.7)$ & $495(5.3)$ \\
\hline Diabetes & $7862(4.7)$ & $2877(4.9)$ & $3125(4.5)$ & $1325(4.5)$ & $463(5.0)$ \\
\hline Transferred to another facility & $14273(8.5)$ & $531(0.9)$ & $697(1.0)$ & $6238(21.0)$ & $6653(71.2)$ \\
\hline Alberta & $18330(10.9)$ & $11739(20.0)$ & $4080(5.9)$ & $634(2.1)$ & $1876(20.1)$ \\
\hline British Columbia & $31660(18.8)$ & $11713(19.9)$ & $12747(18.5)$ & $5404(18.2)$ & $1792(19.2)$ \\
\hline $\begin{array}{l}\text { Northwest, Nunavut or Yukon } \\
\text { Territories }\end{array}$ & $266(0.2)$ & 0 & 0 & $103(0.3)$ & $163(1.7)$ \\
\hline \multicolumn{6}{|l|}{ Day of admission } \\
\hline Weekday & $121857(72.4)$ & $42293(71.9)$ & $49517(72.0)$ & $21722(73.2)$ & $7052(75.5)$ \\
\hline Weekend & $46483(27.6)$ & $16506(28.1)$ & $19226(28.0)$ & $7962(26.8)$ & $2291(24.5)$ \\
\hline \multicolumn{6}{|l|}{ Time of admission§ } \\
\hline 0000-0559 & $23742(14.1)$ & $10844(18.4)$ & $8829(12.8)$ & $2989(10.1)$ & $890(9.5)$ \\
\hline 0600-1159 & $23632(14.0)$ & $7046(12.0)$ & $10653(15.5)$ & $4329(14.6)$ & $1313(14.1)$ \\
\hline $1200-1759$ & $56881(33.8)$ & $17809(30.3)$ & 23249 (33.8) & $11696(39.4)$ & 3496 (37.4) \\
\hline $1800-2359$ & $63987(38.0)$ & $23067(39.2)$ & $25949(37.7)$ & $10669(35.9)$ & $3643(39.0)$ \\
\hline Type of surgeryף & $n=154382$ & $n=54847$ & $n=64054$ & $n=26576$ & $n=7302$ \\
\hline Internal fixation & 92445 (59.9) & $32160(58.6)$ & $38844(60.6)$ & $16006(60.2)$ & $4411(60.4)$ \\
\hline Arthroplasty & $61937(40.1)$ & $22687(41.4)$ & 25210 (39.4) & $10570(39.8)$ & $2892(39.6)$ \\
\hline \multicolumn{6}{|l|}{ Timing of surgery $\|^{* *}$} \\
\hline Admission day or day after & $98532(63.8)$ & $32150(58.6)$ & $45476(71.0)$ & $17271(65.0)$ & 2599 (35.6) \\
\hline$\geq 2 \mathrm{~d}$ after admission & $55845(36.2)$ & $22697(41.4)$ & $18576(29.0)$ & $9305(35.0)$ & $4703(64.4)$ \\
\hline \multicolumn{6}{|c|}{$\begin{array}{l}\text { Note: COPD = chronic obstructive pulmonary disease. } \\
\text { *Does not include } 19 \text { patients with unknown sex. } \\
\text { †At admission; for } 2084 \text { patients with different fracture types at admission and surgery, the fracture type at surgery is presented. } \\
\text { fldentified using diagnosis codes from all hospital admissions in the year before the index admission. } \\
\text { §Does not include } 98 \text { patients with unknown time of admission. } \\
\text { TOnly for patients who underwent surgery. } \\
\text { **Does not include } 5 \text { patients with unknown timing of surgery. }\end{array}$} \\
\hline
\end{tabular}




\section{Results}

\section{Patient and care characteristics}

A total of 168340 patients were admitted with a nonpathological first hip fracture between Jan. 1, 2004, and Dec. 31, 2012 (Figure 1). Most $(72.9 \%)$ were women and almost half $(45.9 \%)$ were 85 years or older. Fracture type was similarly distributed between transcervical $(51.8 \%)$ and trochanteric $(48.2 \%)$ fractures. Overall, $27.9 \%$ of the patients had major comorbidities, with cardiac dysrhythmia being the most prevalent $(9.5 \%)$ (Table 1).

Overall, 58799 (34.9\%) of the patients were admitted to teaching hospitals, and 68743 (40.8\%) were admitted to large, 29684 (17.6\%) to medium and 9343 (5.6\%) to small community hospitals (Table 1); type of hospital was unknown for 1771 patients. More patients admitted to small community hospitals $(71.2 \%)$ were transferred to another facility than were patients admitted to teaching $(0.9 \%)$, large $(1.0 \%)$ or medium $(21.0 \%)$ community hospitals. Admissions between midnight and 0600 were more frequent at teaching hospitals $(18.4 \%)$ than at large $(12.8 \%)$, medium $(10.1 \%)$ or small $(9.5 \%)$ community hospitals. Weekend admissions were more frequent at teaching hospitals $(28.1 \%)$ and large community hospitals $(28.0 \%)$ than at medium $(26.8 \%)$ or small $(24.5 \%)$ community hospitals. More patients in Alberta, Saskatchewan, and New- foundland and Labrador were admitted to teaching hospitals than to large, medium or small community hospitals, compared with patients in other provinces and territories (Table 1).

More patients underwent arthroplasty at teaching hospitals $(38.6 \%)$ than at large $(36.7 \%)$, medium (35.6\%) or small (31.0\%) community hospitals. Of the 154382 patients who underwent surgery, more underwent surgery on admission day or the day after at large community hospitals (66.2\%) than at teaching hospitals (58.6\%) or at medium $(65.0 \%)$ or small $(35.6 \%)$ community hospitals.

\section{In-hospital mortality}

By day 30 after admission, 11672 (6.9\%) hospital stays ended with death, 101817 (60.5\%) ended with live discharge, 26994 (16.0\%) had rightcensoring events, and 27857 (16.6\%) stays were longer than 30 days. The average rate of inhospital death was 4.7 (95\% confidence interval [CI] 4.6-4.7) per 1000 patient-days overall, varying from 4.0 (95\% CI 3.8-4.1) per 1000 patientdays at teaching hospitals, to 4.8 (95\% CI 4.6-4.9), 5.5 (95\% CI 5.3-5.8) and 6.3 (95\% CI 5.8-6.7) per 1000 patient-days at large, medium and small community hospitals, respectively (Table 2).

Compared with the number of deaths per 1000 admissions at teaching hospitals, there were an additional 3 (95\% CI 1-6), 14 (95\% CI 10-18) and 43 (95\% CI 35-51) deaths per 1000 admissions at large, medium and small community

Table 2: Cumulative incidence of death in hospital and death after surgery, by hospital type

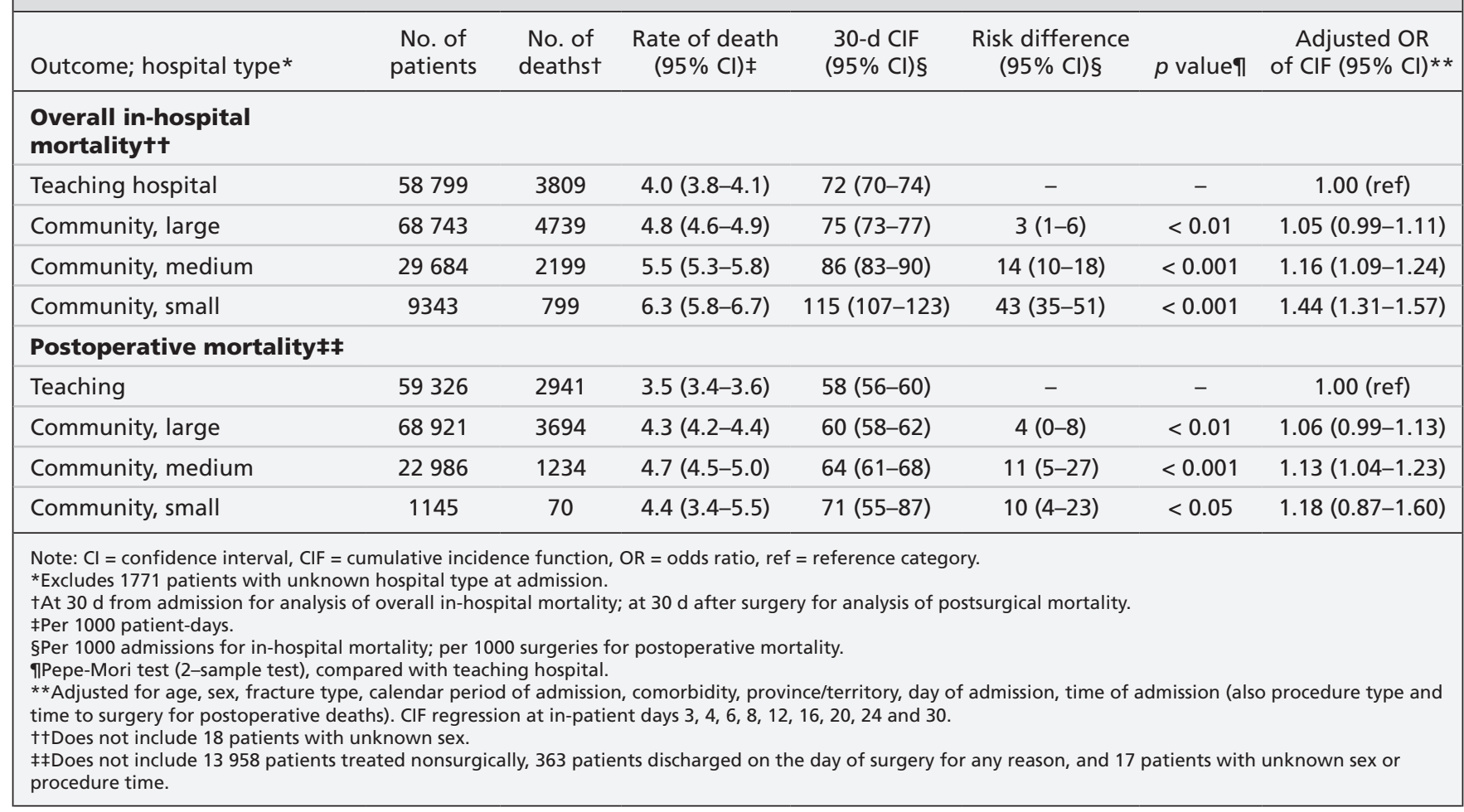


hospitals, respectively (Table 2, Figure 2). For the risk of in-hospital death overall, the adjusted odds ratios (ORs) were 1.05 (95\% CI 0.99-1.11), 1.16 (95\% CI 1.09-1.24) and 1.44 (95\% CI 1.311.57) at large, medium and small community hospitals, respectively, compared with teaching hospitals (Table 2).

\section{Postsurgical mortality}

For this analysis, we included 154019 surgically treated patients after excluding patients who died intraoperatively $(n=237)$ or were discharged alive on the day of surgery $(n=126)$ (Figure 1). By day 30 after surgery, $8035(5.2 \%)$ hospital stays ended with death, 95039 (61.7\%) ended with live discharge, 29324 (19.0\%) had right-censoring events, and $21621(14.0 \%)$ hospital stays were longer than 30 days. The average rate of postsurgical death was 4.0 (95\% CI 3.9-4.1) per 1000 patient-days, varying from 3.5 (95\% CI 3.4-3.6) at teaching hospitals, to 4.3 (95\% CI 4.2-4.4), 4.7 (95\% CI 4.5-5.0) 4.4 (95\% CI 3.4-5.5) at large, medium and small community hospitals, respectively (Table 2).

Compared with the number of deaths per 1000 surgeries at teaching hospitals, there were an additional 4 (95\% CI 0-8), 11 (95\% CI 5-27) and 10 (95\% CI 4-23) deaths per 1000 surgeries at large, medium and small community hospitals, respectively (Table 2). For the risk of postsurgical inhospital death, the adjusted ORs were 1.06 (95\% CI 0.99-1.13), 1.13 (95\% CI 1.04-1.23) and 1.18 (95\% CI 0.87-1.60) at large, medium and small community hospitals, respectively, compared with teaching hospitals (Table 2).

\section{Mortality without surgery}

For this analysis, we included 13958 nonsurgically treated patients. By day 30 after admission, 3649 (26.1\%) died without surgery, 6778 (48.6\%) were discharged without surgery, and 3531 (25.3\%) had right-censoring events. The average rate of death without surgery was 6.3 (95\% CI 6.1-6.5) per 1000 patient-days, varying from 5.3 (95\% CI 5.0-5.6) at teaching hospitals, to 5.9 (95\% CI 5.6-6.2), 7.7 (95\% CI 7.2-8.3) and 8.6 (95\% CI 7.8-9.4) per 1000 patient-days at large, medium and small community hospitals, respectively. Among the 13958 patients treated nonsurgically, the cumulative incidence of death by inpatient day 30 was 19 (95\% CI 18-20) per 1000 admissions at teaching hospitals, and 19 (95\% CI 18-20), 29 (95\% CI 27-31) and 52 (95\% CI 48-57) per 1000 admissions at large, medium and small community hospitals, respectively. Compared with the number of deaths without surgery per 1000 admissions at teaching hospitals, there were an additional 10 (95\% CI 8-12) and 34 (95\% CI 29-39) deaths per 1000 admissions at medium and small community hospitals, respectively. There was no difference between teaching and large community hospitals. The adjusted ORs for death without surgery were 1.02 (95\% CI 0.92-1.14), 1.50 (95\% CI 1.33-1.69) and 2.64 (95\% CI 2.30-3.03) at large, medium and small community hospitals, respectively, compared with teaching hospitals.

\section{Interpretation}

Compared with teaching hospitals, the risk of inhospital death was higher at medium and small community hospitals, and the risk of in-hospital death after surgery was higher at medium community hospitals. The difference in postsurgical mortality between teaching hospitals and small community hospitals, although large, was not significant after adjustment. No differences in outcomes were found between teaching hospitals and large community hospitals.

Our findings are consistent with those from previous reports of increased risk of death among patients treated at community hospitals after hip fracture, ${ }^{9,11,13}$ and among patients treated at hospitals with fewer available beds at admission. ${ }^{31}$ As argued elsewhere, the risk of death in hospital also depends on time spent in hospital, which varies by treatment setting. ${ }^{32}$

We recently showed a reduction in hospital stay after hip fracture following changes in bed management and changes in policy on access to hip fracture surgery in Canada. ${ }^{20}$ How these changes were implemented and how effective they were at reducing hospital stay likely varied by treatment setting. Teaching hospitals may shorten stays more

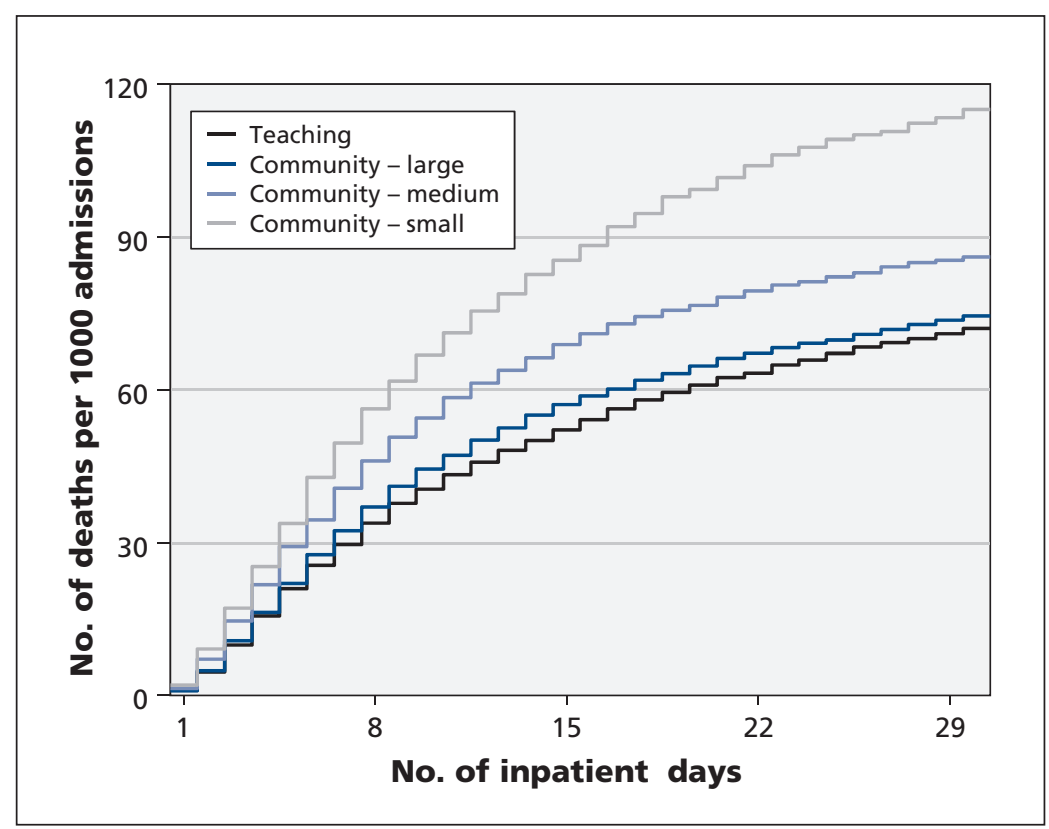

Figure 2: Cumulative incidence of in-hospital death by inpatient days across treatment settings among all patients admitted with first hip fracture. 
effectively because discharge options such as rehabilitation and residential care facilities are more prevalent than in community hospitals. ${ }^{33}$ Our study accounted for this potential bias. In particular, we used the cumulative incidence to estimate the proportion of patients who died in hospital among all patients admitted to hospital with hip fracture while being exposed to the competing risk of live discharge during the follow-up period.

Postsurgical mortality was higher at medium community hospitals than at teaching hospitals. The difference may be attributable to medium community hospitals having fewer beds, staff and equipment available to ensure access to timely hip fracture care,,$^{31,34}$ or to their having a less aggressive treatment style, leaving more patients exposed to potentially fatal immobilized and inflammatory states. ${ }^{1,735-38}$ Whether additional resources for medium community hospitals may improve outcomes in this vulnerable patient population requires further investigation.

The risk of death without surgery was higher at medium and small community hospitals than at teaching hospitals. It is not clear whether this difference reflects a need to transfer patients for specialist care not available at medium and small community hospitals. The time required to transfer patients from medium and small community hospitals for care contributes to potentially harmful surgical delay ${ }^{39}$ It may be necessary to prioritize these patients on arrival at larger hospitals.

Other structures and processes may influence outcomes of hip fracture care. Previous studies have shown an association between a higher volume of hip fracture surgeries and delays, complications and death..$^{40,41}$ The studies suggest underprioritization of hip fracture over other surgeries at high-volume sites. ${ }^{40,41}$ Hospital occupancy has also been associated with risk of in-hospital death after hip fracture. ${ }^{31}$ Future research should explore the association among teaching status, bed capacity, occupancy and volume to better our understanding of outcomes of hip fracture care delivery.

\section{Limitations}

We conducted a secondary analysis of discharge abstracts with limited variables for adjustment. In particular, patients with hip fracture in different treatment settings may differ by pre-fracture function, level of dependency, injury severity, body composition, cognition, and presence of liver disease, anemia, stroke and secondary hyperparathyroidism. ${ }^{42}$ Further, the abstracts do not provide indication for nonsurgical treatment. Palliative care may have been more frequent at medium and small community hospitals. Classification of treatment settings was based on data from the second half of the study period. ${ }^{43}$ This may have led to misclassification of medium and small community hospitals if the number of beds increased across the study years. Bed capacity was not available for teaching hospitals; therefore, we did not investigate difference in mortality by hospital size separately. The hospitals were not identified by their geographic location, which precluded adjustment for urban, rural or remote location. Whether medium and small community hospitals serve more remote populations, or whether Canada's geography could facilitate access to larger hospitals was not factored into our analysis. Few patients underwent surgery at small community hospitals, which, combined with the lack of clinical data, requires some caution in interpretation of the observed differences. Finally, the province of Quebec compiles hospital discharge data in a separate database and does not contribute to the CIHI Discharge Abstracts Database; therefore, the results may not be generalizable to Quebec.

\section{Conclusion}

Compared with teaching hospitals, the risk of inhospital death overall was higher at medium and small community hospitals, and the risk of postsurgical death was higher at medium community hospitals. The difference in postsurgical mortality between teaching hospitals and small community hospitals, although large, was not significant after adjustment. We found no difference between teaching hospitals and large community hospitals. Future research should examine the role of volume, demand and bed occupancy for the observed differences by treatment setting.

\section{References}

1. Uzoigwe CE, Burnand HG, Cheesman CL, et al. Early and ultraearly surgery in hip fracture patients improves survival. Injury 2013;44:726-9

2. Neuhaus V, King J, Hageman MG, et al. Charlson comorbidity indices and in-hospital deaths in patients with hip fractures. Clin Orthop Relat Res 2013;471:1712-9.

3. Hagino T, Ochiai S, Watanabe Y, et al. Hyponatremia at admission is associated with in-hospital death in patients with hip fracture. Arch Orthop Trauma Surg 2013;133:507-11.

4. Penrod JD, Litke A, Hawkes WG, et al. The association of race, gender, and comorbidity with mortality and function after hip fracture. J Gerontol A Biol Sci Med Sci 2008;63:867-72.

5. Vestergaard P, Rejnmark L, Mosekilde L. Loss of life years after a hip fracture. Acta Orthop 2009;80:525-30.

6. Belmont PJ Jr, Garcia EJ, Romano D, et al. Risk factors for complications and in-hospital mortality following hip fractures: a study using the National Trauma Data Bank. Arch Orthop Trauma Surg 2014;134:597-604.

7. Daugaard CL, Jorgensen HL, Riis T, et al. Is mortality after hip fracture associated with surgical delay or admission during weekends and public holidays? A retrospective study of 38,020 patients. Acta Orthop 2012;83:609-13.

8. Forte ML, Virnig BA, Swiontkowski MF, et al. Ninety-day mortality after intertrochanteric hip fracture: Does provider volume matter? J Bone Joint Surg Am 2010;92:799-806.

9. Weller I, Wai EK, Jaglal S, et al. The effect of hospital type and surgical delay on mortality after surgery for hip fracture. J Bone Joint Surg Br 2005;87:361-6.

10. Deiner S, Westlake B, Dutton RP. Patterns of surgical care and complications in elderly adults. J Am Geriatr Soc 2014;62:829-35. 
11. Taylor DH Jr, Whellan DJ, Sloan FA. Effects of admission to a teaching hospital on the cost and quality of care for Medicare beneficiaries. N Engl J Med 1999;340:293-9.

12. Alzahrani K, Gandhi R, Davis A, et al. In-hospital mortality following hip fracture care in southern Ontario. Can J Surg 2010; 53:294-8

13. Yuan Z, Cooper GS, Einstadter D, et al. The association between hospital type and mortality and length of stay: a study of 16.9 million hospitalized Medicare beneficiaries. Med Care 2000;38:231-45.

14. Bazzoli GJ, Brewster LR, May JH, et al. The transition from excess capacity to strained capacity in U.S. hospitals. Milbank $Q$ 2006;84:273-304.

15. Menzies IB, Mendelson DA, Kates SL, et al. Prevention and clinical management of hip fractures in patients with dementia. Geriatr Orthop Surg Rehabil 2010;1:63-72.

16. Technical notes for analyses of hip fracture admissions. Ottawa: Canadian Institute for Health Information; 2005. Available: https:// secure.cihi.ca/free_products/WaitTimesReport_tech_Hip_e.pdf (accessed 2016 Oct. 5)

17. Bohm E, Loucks L, Wittmeier K, et al. Reduced time to surgery improves mortality and length of stay following hip fracture: results from an intervention study in a Canadian health authority. Can J Surg 2015;58:257-63.

18. Data quality documentation for external users: Discharge Abstract Database, 2010-2011. Ottawa: Canadian Institute for Health Information; 2014

19. Sheehan KJ, Sobolev B, Guy P, et al. Constructing an episode of care from acute hospitalization records for studying effects of timing of hip fracture surgery. J Orthop Res 2016:34:197-204.

20. Sobolev B, Guy P, Sheehan KJ, et al. Time trends in hospital stay after hip fracture in Canada, 2004-2012: database study. Arch Osteoporos 2016;11:13.

21. Peer groups in the electronic Discharge Abstract Database reports. Ottawa: Canadian Institute for Health Information; 2015.

22. Pepe MS, Mori M. Kaplan-Meier, marginal or conditional probability curves in summarizing competing risks failure time data? Stat Med 1993;12:737-51.

23. Klein JP, Andersen PK. Regression modeling of competing risks data based on pseudovalues of the cumulative incidence function. Biometrics 2005;61:223-9.

24. Relative risk, risk differences and odds ratio. Ostend (Belgium): MedCalc. Available: www.medcalc.org/manual/relativerisk_ oddsratio.php (accessed 2015 July 24).

25. Nikkel LE, Fox EJ, Black KP, et al. Impact of comorbidities on hospitalization costs following hip fracture. J Bone Joint Surg Am 2012;94:9-17.

26. Auais M, Morin S, Nadeau L, et al. Changes in frailty-related characteristics of the hip fracture population and their implications for healthcare services: evidence from Quebec, Canada. Osteoporos Int 2013;24:2713-24.

27. Ban I, Palm H, Birkelund L, et al. Implementing, adapting, and validating an evidence-based algorithm for hip fracture surgery J Orthop Trauma 2014;28:e21-6.

28. Gray B. cmprsk: subdistribution analysis of competing risks 2014. Available: http://CRAN.R-project.org/package $=\mathrm{cmprsk}$ (accessed 2015 July 24)

29. Gerds T. prodlim: Product-limit estimation for censored event history analysis; 2014. Available: http://CRAN.R-project.org/ package $=$ prodlim (accessed 2015 July 24).

30. Hojsgaard S, Halekoh U, Yan J. The R package geepack for generalized estimating equations. J Stat Softw 2006;15:1-11.

31. Schilling PL, Campbell DA Jr, Englesbe MJ, et al. A comparison of in-hospital mortality risk conferred by high hospital occupancy, differences in nurse staffing levels, weekend admission, and seasonal influenza. Med Care 2010;48:224-32.

32. Nordström P, Gustafson Y, Michaelsson K, et al. Length of hospital stay after hip fracture and short term risk of death after discharge: a total cohort study in Sweden. BMJ 2015;350:h696.

33. Williams N, Hardy BM, Tarrant S, et al. Changes in hip fracture incidence, mortality and length of stay over the last decade in an Australian major trauma centre. Arch Osteoporos 2013;8:150.

34. Silber JH, Kaestner R, Even-Shoshan O, et al. Aggressive treatment style and surgical outcomes. Health Serv Res 2010;45:1872-92.

35. Beloosesky Y, Grinblat J, Pirotsky A, et al. Different C-reactive protein kinetics in post-operative hip-fractured geriatric patients with and without complications. Gerontology 2004;50:216-22.

36. Beloosesky Y, Hendel D, Weiss A, et al. Cytokines and C-reactive protein production in hip-fracture-operated elderly patients. J Gerontol A Biol Sci Med Sci 2007;62:420-6.

37. Koval KJ, Rust CL, Spratt KF. The effect of hospital setting and teaching status on outcomes after hip fracture. Am J Orthop 2011;40:19-28

38. Peleg K, Savitsky B, Yitzhak B, et al. Different reimbursement influences surviving of hip fracture in elderly patients. Injury 2011;42:128-32

39. Zeltzer J, Mitchell RJ, Toson B, et al. Determinants of time to surgery for patients with hip fracture. ANZ J Surg 2014;84:633-8.

40. Kristensen PK, Thillemann TM, Johnsen SP. Is bigger always better? A nationwide study of hip fracture unit volume, 30-day mortality, quality of in-hospital care, and length of hospital stay. Med Care 2014;52:1023-9.

41. Metcalfe D, Olufajo OA, Zogg CK, et al. Are older adults with hip fractures disadvantaged in level 1 trauma centers? Med Care 2016;54:616-22.

42. Sheehan KJ, Sobolev B, Chudyk A, et al. Patient and system factors of mortality after hip fracture: a scoping review. $B M C$ Musculoskelet Disord 2016;17:166.

43. CIHI Portal release note: release 9.14. Ottawa: Canadian Institute for Health Information; 2015

Competing interests: Pierre Guy receives funding from the Natural Sciences and Engineering Research Council of Canada, the Canadian Foundation for Innovation and the British Columbia Specialists Services Committee for work about hip fracture care not related to this study; he has received fees from the BC Specialists Services Committee (for a provincial quality improvement project on redesign of hip fracture care) and from Stryker Orthopedics (as a product development consultant); he is a board member and shareholder in Traumis Surgical Systems Inc. and a board member for the Canadian Orthopedic Foundation; and he serves on the speakers' bureaus of AO Trauma North America and Stryker Canada. Suzanne Morin has received research grants from Amgen Canada and Merck, and consultation fees from Amgen Canada outside the submitted work. Katie Sheehan is a postdoctoral fellow whose salary is paid by the Canadian Institutes of Health Research funding related to this work.

Affiliations: School of Population and Public Health (Sheehan, Sobolev, Sutherland) and Department of Orthopedics (Guy), University of British Columbia, Vancouver, BC; Centre for Clinical Epidemiology and Evaluation (Kuramoto), Vancouver Coastal Health Research Institute, Vancouver, BC; Department of Medicine (Morin), McGill University, Montréal, Que.; Department of Physical Therapy and Division of Orthopaedic Surgery (Beaupre), University of Alberta, Edmonton, Alta.; Department of Anesthesiology, Pharmacology and Therapeutics (Griesdale), University of British Columbia, Vancouver, BC; Division of Orthopaedic Surgery (Dunbar), Dalhousie University, Halifax, NS; Division of Orthopaedic Surgery and Centre for Healthcare Innovation (Bohm), University of Manitoba, Winnipeg, Man.; Division of Orthopaedic Surgery (Harvey), McGill University, Montréal, Que.

Contributors: All of the authors contributed to the conception and design of the study. Katie Sheehan, Boris Sobolev, Pierre Guy and Lisa Kuramoto contributed to the acquisition and analysis of data. All of the authors contributed to the interpretation of the analysis. Katie Sheehan and Boris Sobolev drafted the manuscript. All of the authors critically revised the manuscript, approved the final version to be published and agreed to act as guarantors of the work.

Funding: This study was funded by the Canadian Institutes of Health Research (grant no. MOP-133629). The funding body had no role in the design or execution of the study, the analysis or interpretation of the data, or the decision to submit the manuscript for publication.

Acknowledgements: The authors acknowledge the guidance from the experts at the Canadian Institute for Health Information in understanding the discharge abstracts. They also thank Nick Bradley, Kirill Gordin and Michael Tang for preparing data, and Janet Lam for generating figures for the manuscript.

Members of the Canadian Collaborative Study of Hip Fractures: Eric Bohm, Lauren Beaupre, Michael Dunbar, Donald Griesdale, Pierre Guy, Edward Harvey, Erik Hellsten, Susan Jaglal, Hans Kreder, Lisa Kuramoto, Adrian Levy, Suzanne N. Morin, Katie J. Sheehan, Boris Sobolev, Jason M. Sutherland and James Waddell. 